\title{
CANTORIAN INFINITY AND PHILOSOPHICAL CONCEPTS OF GOD
}

\section{JOANNA VAN DER VEEN \& LEON HORSTEN}

\author{
University of Bristol
}

\begin{abstract}
It is often alleged that Cantor's views about how the set theoretic universe as a whole should be considered are fundamentally unclear. In this article we argue that Cantor's views on this subject, at least up until around 1896, are relatively clear, coherent, and interesting. We then go on to argue that Cantor's views about the set theoretic universe as a whole have implications for theology that have hitherto not been sufficiently recognised. However, the theological implications in question, at least as articulated here, would not have satisfied Cantor himself.
\end{abstract}

\section{INTRODUCTION ${ }^{1}$}

In Cantor's philosophy of mathematics a connection is made between the mathematical universe $e^{2}$ considered as a whole (the quantitative Absolute Infinite) and God. In this article we aim to investigate whether this contention is correct.

We will start by considering Cantor's ontology and epistemology of sets, and the ways in which he felt that these were connected to the nature of God's mind. Cantor essentially takes elements of Augustine's view as a point of departure, and develops them in a distinctive and innovative way. Parallel to this, we will investigate the characteristics of God

\footnotetext{
${ }^{1}$ Thanks to Ignacio Jané and Christian Tapp for insightful comments.

${ }^{2}$ In this paper, 'mathematical universe' and 'set theoretic universe' are interchangeable, as we are working from the viewpoint that set theory is foundational for mathematics. As such, any observation we make about the set theoretic universe can also be made about the mathematical universe.
} 
according to Cantor; similarities and connections between the two will become evident. This will then allow us to develop a Cantorian argument that derives theological conclusions from reasonable assumptions about the mathematical universe considered as a whole. Crucially, however, this argument is not Cantor's own. It relies on non-19th century premises. In particular, the argument uses naturalistic assumptions about the nature of mathematical objects: it assumes certain 'naïve' commitments and beliefs that are implied by the practice of working mathematicians. When these are combined with more Cantorian elements (in particular, the existence of the set theoretic universe as a completed whole), significant theological implications can be derived; however, these implications fall short of establishing the existence of a personal God. To conclude, we will offer a defence of some of the assumptions crucial to the narrative namely, those of naturalism and our distinctive conception of the set theoretic universe as a completed whole.

An important caveat must be made before beginning. We refer to 'God' throughout the article, but do not intend the term to be understood in the traditional theistic sense (unless otherwise implied by the context). Instead, we use the term loosely, allowing for the possibility of different sorts of religious perspectives - for example, deism or even pantheism. So, broadly speaking, we use the term to indicate something supernatural or 'divine'.

\section{CANTOR'S MATHEMATICAL ONTOLOGY AND EPISTEMOLOGY}

In this section, we consider Cantor's mathematical ontology and its related epistemology. In his writings, Cantor rarely clearly delineated his theological commitments from his mathematical ones and, as such, the following picture might appear obscure to many mathematicians. However, an understanding of how Cantor conceived of the mathematical universe will inform the (somewhat modernised) discussion that follows.

Kowalewski once commented that for Cantor the infinite cardinal numbers seemed 'stepping stones to the throne of God' (Kowalewski 1950: 201). Indeed, Cantor writes that God lies 'beyond the cardinal numbers' (Letter to Grace Chisholm-Young (1908), Cantor 1991: 454). The Cantorian picture seems to be one of the cardinal numbers converging to a limit that lies beyond themselves, with that limit conceived of as 'God'. It is clear that Cantor thought that God could not be investigated 
mathematically, being instead the subject of 'speculative theology' (Cantor 1932: 378). Therefore, the many remarks on the nature of God that one finds in Cantor's mathematical-philosophical texts suggest that, whilst his notion of God is somehow connected with his theory of the transfinite and cardinal numbers, it is at the same time, at least to some extent, disconnected from mathematics.

Cantor was a Lutheran with deep sympathies for the Catholic church, and his conception of the nature of God (which will be considered in more detail in section 3 ) is tied to the Christian philosophicaltheological tradition. On this subject, he primarily quotes philosophers and theologians, and this can lead to the impression that in this area Cantor had few original thoughts. This impression is unjustified, as we will see shortly.

Although Cantor held that the nature and properties of God cannot be investigated mathematically, his views on God were of crucial importance for his defence of his theory of the transfinite; he used his conception of God to motivate his conception of infinity in mathematics (specifically in set theory).

Cantor upheld the Augustinian view that mathematical entities such as numbers exist as ideas in the mind of God (Letter to Jeiler (1895), Tapp 2005: 427, our translation):

The transfinite is capable of manifold formations, specifications, and individuations. In particular, there are transfinite cardinal numbers and transfinite ordinal types which, just as much as the finite numbers and forms, possess a definite mathematical uniformity, discoverable by men. All these particular modes of the transfinite have existed from eternity as ideas in the Divine intellect.

Like Augustine (Augustine 2003: Ch. 18), he thought that the collection of the natural numbers is 'in a certain sense' finite for, and thus knowable by, God. However, Cantor extended Augustine's underdeveloped view in a revolutionary way. On the mathematical and epistemological side, Cantor claimed that the transfinite is knowable not only by God, but also by humans. Cantor's transfinite set theory shows how human mathematicians are able to calculate with transfinite numbers. On the philosophical and ontological side, Cantor developed Augustine's sketchy view in a very original way. In a remarkable passage, Cantor articulates in detail how sets exist in the mind of God. The passage in question gives us a unique insight into Cantor's ontology and epistemology of 
mathematics. We quote him here in full (Tapp 2005: 414-407, Letter to Jeiler (1888), our translation):

God knows infinitely many things in reality and categorematically outside Himself (possible objects, objects that occur throughout the time series in the future), that admittedly do not always occur together on the side of the things, but that do have a simultaneity in their being known in God's Intellect. If only one would have convinced oneself always of this secure and unshakeable proposition in its full content (I mean, not just in general, but also in special and concrete cases), then one would have recognised in it without any trouble the truth of the transfinite, and millennia of disputes and errors would have been avoided.

If we now apply this proposition to a special class of objects of God's intellect, then we arrive at the elements (elementary propositions) of the theory of infinite numbers and order types.

Every single finite cardinal number ( $\underline{1}$ or $\underline{2}$ or $\underline{3}$, etc) is contained in God's Intellect both as an exemplary idea, and as a unitary form of knowledge of countless compound objects, to which the cardinal number applies: all finite cardinal numbers are therefore distinctly and simultaneously present in God's mind (Augustine 2003: book XII, chapter 19: 'Against those, who say that, what is infinite, can also not be comprehended by God's knowledge'). ${ }^{3}$

They build in their totality a manifold, unified, and from the other contents of God's Intellect separated thing in itself, that itself forms an object of God's Knowledge. But since the knowledge of a thing presupposes a unitary form, by which this thing exists and is known, there must in God's intellect be a determinate cardinal number available, which relates itself to the collection or totality of all finite cardinals in the same way as the number 7 relates itself to the notes in an octave.

For this, which can be shown to be the smallest transfinite cardinal number, I have chosen the sign $\underline{\omega}$.

On the other hand the finite cardinal numbers $\underline{1}, \underline{2}, 3, \ldots$ form in their natural order a well-ordered collection [...]; the general form, under which this well-ordered collection of all finite cardinal numbers is necessarily conceived by God's Intellect (on reflection on them

${ }^{3}$ Cantor is in fact referring to the content of chapter 18 rather than of chapter 19 here. 
belonging to the ordering that I have just described), I call the ordinal number of this well-ordered collection, or its order type, and I signify it with $\omega$; here, too, it can easily be shown that $\omega$ is the smallest transfinite ordinal number.

When we abstract in $\omega$ from the ordering of its elements (which are then just units), then we will naturally obtain the cardinal number that we have denoted above by $\underline{\omega}$.

This is to explain my notation; the bar over ${ }^{4}$ the $\omega$ should remind of the abstraction from the ordering of the elements in the cardinal number $\underline{\omega}$; one can say, that the cardinal number $\omega$ originates from the ordinal number $\omega$, when we abstract in the latter from the ordering of the units that are contained in it.

The picture that emerges is the following. Finite cardinal numbers, and even 'small transfinite numbers' such as $N_{0}$ and $N_{1},{ }^{5}$ are obtained by a process of abstraction from groupings in the physical world. This process of abstraction should not be conceived of as a human form of creation; instead, humans discover numbers as abstracta from groupings that have existed for all eternity in the mind of God. The same holds for ordinal numbers. They exist in the mind of God as abstracta of configurations in the world under the aspect of an ordering relation. When humans have knowledge of sets, they stand in cognitive contact with these mathematical forms in the mind of God. ${ }^{6}$

Cantor does not claim that cardinalities larger than $N_{1}$, are instantiated in the material world, and consequently it cannot be assumed that all cardinalities exist in God's mind as abstractions from groupings of material entities in the world. ${ }^{7}$ But if the natural numbers themselves form a set in the mind of God, and all subcollections of natural numbers likewise form sets in the mind of God, then these entities themselves form a well-delineated totality in the mind of God, from which a number can then be abstracted $\left(2^{\mathrm{No}}\right)$. We can then repeat this process; in this way, all cardinalities (finite and transfinite) are instantiated in the mind of God, and so exist as numbers in the mind of God.

${ }^{4}$ We have used bars under the digits instead of above them; the meaning is the same

${ }^{5}$ Cantor believed that the material atoms form a countably infinite set, and that the ether particles form a smallest uncountable set (Cantor 1991: 224, Letter to MittagLeffler (1884)).

${ }^{6}$ Benacerraf (1973) asks searching questions about the nature of this cognitive relation. However, a discussion of this problem exceeds the scope of the current article.

${ }^{7}$ Similarly for ordinal numbers. 
This conception of Cantor's ontology of sets helps to elucidate another comment made in the above passage, viz. that cardinal and ordinal numbers exist in God's mind in two ways: as unitary forms and as exemplary ideas. ${ }^{8}$ This 'double existence' of mathematical entities in God's mind can potentially be understood in two ways:

(1) Numbers exist as two different entities in the mind of God.

(2) They exist as only one entity, but this entity has two different aspects.

Cantor seems to lean towards the first option in the quoted passage. Under this conception, the number one, for instance, exists as a 'unitary form' that is an abstractum from concrete unities in the world. But this unitary form can itself be seen as a very special singleton: the singleton of the number one. The latter object is an exemplary idea, a special instantiation of the unitary form. Similarly, the number 2 is instantiated in a very special idea in the mind of God as the set $\{1,2\}$. The ordinal $\omega$ exists in an exemplary way as the ordered sequence $\langle 1,2, \ldots\rangle$, and when we abstract from the ordering we obtain the exemplary way in which the first infinite cardinal number exists. Continuing in this way, the ordinal number $\omega+\omega$ is instantiated in a very special way as the ordered sequence $\langle 1,2, \ldots \omega, \omega+1, \ldots\rangle$, and so on. In general, Cantor's two generating principles guarantee the exemplary existence of all ordinal and cardinal numbers in the mind of God. ${ }^{9}$ Observe that, of course, the ordinal number $\omega+\omega$ also exists in 'non-exemplary ways' - for instance as the ordered sequence $\langle\omega, \omega+1, \ldots 1,2, \ldots\rangle$.

All these mathematical 'forms' taken together form the quantitatively Absolutely Infinite. This follows from an unrestricted fusion principle (not mentioned by Cantor) which says that any plurality of objects taken together form a compound object (Niebergall 2012: 277). In the metaphysical literature, this principle is mostly applied to concrete objects, but it can likewise be applied in the abstract realm. Metaphysicians tend to assume unrestricted fusion for objects ${ }^{10}$ and - as we are considering sets as objects - we do not find it unreasonable to apply the same such principles to our own enterprise. The compound object made up by all the mathematical forms must then be 'the biggest infinity'.

\footnotetext{
8 Thanks to Ignacio Jané for helping us understand this aspect of Cantor's view.

${ }^{9}$ For a discussion of Cantor's generating principles, see (Hallett 1984: chapter 2, section 2.1).

10 See (Niebergall 2012: 276).
} 
Nonetheless, as far as Cantor is concerned, all the mathematical forms cannot together form one well-delineated totality in the mind of God from which a number can be abstracted. Cantor does not philosophically spell out why this is so, but he rightly states that he never claimed that a number can be assigned to the collection of all cardinal (or ordinal) numbers. Of course, later in his life Cantor did know why, at least in a mathematical sense - it is a consequence of Russell's Paradox, i.e., of Cantor's own diagonal argument. However, this mathematical insight does not have a parallel philosophical-theological one - for, from the Augustinian point of view, it does seem that the set theoretic universe constitutes a (well-delineated) part of God's mind. As such, the Quantitative Absolute Infinity of the complete set theoretic universe does not appear to sit completely comfortably within Cantor's metaphysical picture.

What Cantor should have said (but did not) on this last point is the following. The mathematical realm is indeed a well-delineated part of the mind of God. But there is much else beside sets in the mind of God. Thus the question of in what sense the quantitatively Absolutely Infinite is the 'biggest infinity' indeed arises. The quantitative extent of the infinity of God is expressed exhaustively in the mathematical part of the mind of God and so, in agreement with von Neumann's principle that all proper classes are 'equally large', the whole of God's mind is in one-to-one onto correspondence with its mathematical part. Thus the set theoretic universe (V) is indeed the 'biggest infinity', and we have some philosophical understanding of why that is so.

An underlying assumption that has been made throughout the foregoing discussion is that the mathematical universe is unique - in other words, that when we speak of the 'mathematical universe' (equivalently, the set theoretic universe) there is exactly one thing that lies within the extension of such a description. Though this is an arguably contentious point in modern philosophy of mathematics, it is outside the scope of the present topic, and as such we will not discuss it further here. ${ }^{11}$

\section{CHARACTERISTICS OF GOD ACCORDING TO CANTOR}

It is blindingly obvious from the preceding discussion that Cantor saw a connection between his work on infinity and his concept of God. We

${ }^{11}$ For one form which this discussion can take, see the debate between (Balaguer 1995) and (Martin 2001). 
will now consider several specific characteristics that Cantor conceived of God as having that are distinct from the above and which, if accepted, will form an independent motivation for the stipulation of a connection between set theory and theology. This sort of exposition has already been done fruitfully by Christian Tapp (see (Tapp 2005) and (Tapp 2012)) and also to some extent by Adam Drozdek (see Drozdek 1995), and we draw heavily on their work here.

Cantor's divine attributes include absolute freedom (Letter to Franzelin (1886), Cantor 1991: 258; Cantor 1932: 406), absolute goodness (Tapp 2005: 326), absolute omnipotence (Tapp 2005: 326) and absolute wisdom (Tapp 2005: 326). 'Absolute' here could (and we suggest should) be read as having a more technical definition than commonly used, one that is understood by considering its Latin roots. Tapp does this in (Tapp 2012: section 2). 'Absolute' in Latin is absolutum, which comes from the root absolvere, meaning to detach or disassociate. So, in essence, what predicating a quality (such as freedom) with 'absolute' does (for Cantor) is detach it from limitations. This might seem superfluous for the divine attributes - after all, is not the very nature of 'freedom' to be detached from limitations? However, he really does mean something stronger here; whilst describing a human as 'free' might simply denote that person's autonomy regarding their life decisions (to marry who they like, for example), when it is applied (absolutely) to God it means that He can literally do whatever He likes - even transcend the laws of physics (something impossible for a human).

When explicated in this way, it becomes clearer why there might be a connection between theological questions and infinity. Infinity is, in a sense, beyond limitations. Pre-theoretical notions of infinity tend to be of the point beyond which we can no longer 'count'; it is outside the limits of the mathematical objects we can sensically work with. Of course, much of advanced mathematics does work with infinity, and Cantor's mathematics even involves completed infinities (for example, transfinite numbers). However, we argue that there is nonetheless a parallel with this pre-theoretic notion of 'beyond limitations', and this is due to Cantor's absolutely infinite (informally, the 'biggest' infinity). ${ }^{12}$ The absolutely infinite is limited only by itself - it is the domain of all other infinities and is thus in a sense a fixed object that is nonetheless unboundedly large.

${ }^{12}$ For a discussion of the relation between Cantor's notion of Absolute and the use of the term 'Absolute' in the history of metaphysics, see (Hauser 2011). 
God, as sketched above, is a 'being' of fixed characteristics, but each of these he possesses in unbounded and yet maximum measure. The only restrictions that can be placed on absolute infinity or on God come from themselves. The similarity between these two conceptions provides some motivation for attempting to uncover a parallel or connection between Cantorian infinities and some notion of God (Cantorian or otherwise).

Another attribute of God which we can arguably extrapolate from Cantor's conception, and which will become crucial later, is that God is beyond human understanding:

I have never assumed a 'Genus Supremum' of the actual infinite. Quite on the contrary I have proved that there can be no such 'Genus Supremum' of the actual infinite. What lies beyond all that is finite and transfinite is not a 'Genus'; it is the unique, completely individual unity, in which everything is, which comprises everything, the 'Absolute', for human intelligence unfathomable, also that not subject to mathematics, unmeasurable, the 'ens simplicissimum', the 'Actus purissimus', which is by many called 'God'. (Letter to Grace Chisholm-Young (1908), Cantor 1991: 454, our translation). ${ }^{13}$

An independent and informal argument for the thesis that God exceeds human comprehension runs as follows. If God were understood by humans, then He would be limited by something outside Himself. From the preceding paragraph we know that God is only limited by Himself. Therefore, God could not possibly be understood by humans. The first premise of this argument requires support. Humans are outside of God (because they are created), and are also finite in their capacity of understanding. Therefore their intelligence (i.e. what they can understand) has an upper bound. So if we were to understand God, then he would have to 'lie within' this boundary. This would be a form of limitation, which, if we accept the foregoing, is impossible. So, in sum, the absoluteness of God's infinity is beyond our understanding.

So transcending human understanding can be taken to be a distinctive feature of God; this both squares with what we know about Cantor's view and is independently compelling (if one is already sold on the idea of

\footnotetext{
${ }^{13}$ Another passage that conveys the same message is the following: 'The Transfinite points [...] with necessity to an Absolute that cannot in any way be diminished, and that is therefore to be looked upon quantitatively as an absolute maximum. In a certain sense it transcends human power of comprehension, and in particular is beyond mathematical determination.' (Cantor 1932: 405)
} 
a divine entity). This particular attribute is also given further support by the fact that it is traditionally taken to be a fundamental property that sets God apart from everything else in creation (negative theology).

Section 2 of this paper has already suggested Cantor's own version of this view - that the set theoretic universe as a whole $(\mathrm{V})$ is a part of God that is too vast for human comprehension (and if a part is incomprehensible, then it stands to reason that the whole is too). However, he did qualify this to some extent. Whilst repeatedly stressing that the Absolutely Infinite exceeds 'mathematical determination', he leaves room for the hypothesis that another kind of understanding of the Absolutely Infinite can be obtained. As mentioned before, he says that the investigation of the Absolutely Infinite is the province of 'speculative theology'. We will have more to say later about Cantor's concept of speculative theology. Let it suffice for now to note that according to the standard view theology differs from philosophy in that it takes the Scripture as given in much the same way as empirical science takes empirical observations and the outcomes of experiments as given.

We argue that, as such, the possibility of understanding the divine through speculative theology is not a threat to our claim that the divine is fundamentally outside the remit of human understanding. The support for this claim has two parts. Such a form of understanding takes the Scripture (or some other sort of religious basis) as given, whereas we are aiming to take nothing 'theological' as given, and to work towards a theological conclusion using only the rules of reason and acceptable premises. Therefore the potential insight given by speculative theology is distinct from the sort of insights that our current conception of 'human understanding' might include. Furthermore, as we have already mentioned, theology and science have distinct methodologies, and so lead to different forms of understanding; in this paper, we are concerned with the sort of understanding given by the latter. Consequently, even if Cantor is correct in his claim that God can be understood (to some extent, and in some specific sense) through speculative theology, we are still legitimately able to claim that a distinctive feature of God is being beyond human understanding.

\section{THE SET THEORETIC UNIVERSE AND THE EXISTENCE OF GOD}

In this section, we bring together the ideas we have outlined so far in order to construct an argument (which will be split into two subsidiary 
arguments) to the conclusion that some sort of divine entity exists. We again stress that such an argument is not Cantor's own; crucially, it falls short of establishing a Christian God - or in fact any sort of personal God. We will endeavour to assess both the arguments and whether the conclusion that they might establish, mitigated as it is, is still significant.

\subsection{Two Arguments}

Before combining the ingredients that we have discussed to construct an argument for the existence of God, we derive a subsidiary conclusion that the mathematical universe is an aspect of God. This, combined with some assumptions about set theoretic ontology and mereology, will be sufficient to create a valid argument; soundness will be investigated afterwards. Very roughly, the proposed reasoning for the subsidiary conclusion goes as follows:

Premise 1 Anything that exceeds human understanding is an aspect of God.

Premise 2 The mathematical universe exceeds human understanding.

Conclusion The mathematical universe is an aspect of God.

If this argument is accepted then, if we can also conclude that the mathematical universe exists, we can conclude that some sort of God exists (because part of God does). We now argue that the mathematical universe does exist:

Premise 1'Sets exist (in a platonic sense)

Premise 2' If a plurality of entities (such as sets) exist, then taken together they form a completed (mereological) whole

Conclusion' The set theoretic universe (equivalently, the mathematical universe) exists

We take it for granted that this argument is valid - as we have taken the set theoretic universe to be nothing but the fusion of all individual sets (see the end of section 2). In the next section, we turn to a consideration of the soundness of both arguments, denoting the first 'Argument 1' and the second 'Argument 2'. We will neither unconditionally endorse nor reject the premises of these two arguments; we shall merely argue that each of them has received enough support in the literature for it to be unreasonable to reject the arguments out of hand as unsound. 


\subsubsection{Argument 2}

Premise 1' is an extremely contentious point in the philosophy of mathematics. A thorough consideration of it is beyond the scope of this article - books could, and indeed have, been written both in defence and in criticism of mathematical platonism. For our current purposes we shall content ourselves with saying that, in light of the naturalist stance that we have chosen to adopt, such a premise is supported by mathematical practice; as Bernays says, every working mathematician is a platonist (Bernays 1935). So mathematical practice, combined with our specific sort of naturalism, provides ample support for this premise.

We have remarked before that Premise 2', on the other hand, is a mereological claim that is widely held in metaphysical circles and, as such, is not greatly controversial. ${ }^{14}$ Indeed, the completed existence of the whole of the set theoretic universe supervenes on the completed existence of the components (in this case, the sets).

However, both these premises are susceptible to criticism, especially if a naturalistic position (in the philosophy of maths) is rejected. After all, it is coherent to reject the claim that our best mathematical ontology can be read off from what mathematicians commit themselves to in their practice. Mathematical naturalism can even be accepted whilst denying the premises in question. However, if this line of criticism were adopted, it would follow that the commitments of mathematicians are not straightforwardly readable from their practice and, as such, that the surface structure of informal mathematical speech is not the same as its deep logical structure. ${ }^{15}$ This in itself constitutes a mark against such a position. Moreover, such a view creates a host of other problems for the philosopher of mathematics; ${ }^{16}$ for the purposes of this article, we will take this as substantial grounds for retaining naturalism and Premise 1.

However, it is also possible to accept the first premise but reject the second; for example, Zermelo conceived of the set theoretic universe as a potentially infinite series of actually infinite 'normal domains' (Zermelo 1930). It has even been argued that from 1896 onwards Cantor himself was well on his way to Zermelo's viewpoint (see, for example, Jané 1995). ${ }^{17}$ This is often taken to be a more sophisticated position than

${ }^{14}$ David Lewis has famously championed the principle of unrestricted fusion that entails it. Nonetheless, his view has been challenged by van Inwagen (van Inwagen 1994).

${ }^{15}$ See, for example, Hellman's position in (Hellman 1989).

${ }^{16}$ For a discussion of this, see (Burgess and Rosen 1999). 
that of Cantor but, again, adopting this position involves not taking set theoretic practice completely at face value. Mathematicians (set theorists) do talk about $\mathrm{V}$ as if it is an entity.

Cantor himself thought (in typical 19th century vein) that the second premise could be proved from prior grounds. His so-called 'domain principle' states that every variation of a parameter presupposes an underlying fixed domain over which the parameter varies (Hallett 1984: 7). Since in Zermelo's picture the 'normal domains' somehow vary ('grow'), there must be a completed domain over which they do this. This domain is, of course, the completed quantitatively Absolutely Infinite $(\mathrm{V})$. These points will be returned to in greater detail later. But it is important to observe that, given naturalism, we need not commit ourselves to the domain principle for the argument to carry through.

Taking these two premises together, we get to the conclusion that the set theoretic universe (V) exists as a completed whole. This conclusion, aside from being the result of a valid and arguably sound argument, is also supported by mathematical practice. If one takes set theoretic practice at face value, then there does seem to be a commitment to $\mathrm{V}$ and other proper classes as completed wholes. After all, many (perhaps most) set theory textbooks contain class forming operations of the form $\{\mathrm{x} \mid \phi(\mathrm{x})\}^{18}$ - it is just that they are very careful about the way that they are handled! In informal argumentation, set theorists have no compunction at all about speaking of the class of the ordinals, for instance. In sum, it is fair to say that we can rely on mathematical practice to assume that, for any definable condition of sets, there is a class (but crucially, not necessarily a set) corresponding to it containing those sets that meet the condition in question.

\subsubsection{Argument 1}

We now turn to the soundness of Argument 1. Premise 2 has been established to some extent by the initial part of this article, but still requires some additional elucidation and support.

The set theoretic universe is known to be beyond the grasp of set theoretic principles as a result of Cantor's diagonal lemma. However, this is not enough to conclude that it is beyond the grasp of human understanding. In order to establish such a thing, we would need to

17 Jané later qualified this judgement: see (Jané 2010).

${ }^{18}$ See for instance (Drake 1974: 3). 
assume that the set theoretic universe's governing principles are those of set theory, which we will take to be the principles of Zermelo-Fraenkel set theory with the Axiom of Choice (ZFC). Thus we need to support the implicit assumption that such a set theory is our most general rational theory of pluralities of sets (as we are conceiving of the set theoretic universe as a plurality of its constituent parts - namely, the plurality of all sets). The claim is essentially that, if some pluralities are not subject to the principles of ZFC, then these collections cannot really be rationally understood.

This is not entirely compelling. We have already seen that Cantor thought it possible that some non-mathematical insight into $\mathrm{V}$ as a completed Absolutely Infinite was possible. However, he was completely silent about what the nature of such an insight could be, and even suggested it was as much a matter of revelation as of real understanding a matter which we have already discussed in section 3. A more threatening challenge to the assumption that ZFC is the best theory we have to understand $\mathrm{V}$ is to propose an alternative theory which does give us a mathematical understanding of proper classes: Von NeumannBernays-Gödel set theory (NBG). ${ }^{19}$ However, such a claim could be challenged by replying that NBG does not really give us a treatment of proper classes as entities; it is no more than an acknowledgement that to every condition of sentences, a class corresponds that contains precisely those sets as elements that meet the condition. So NBG does not give us a mathematical understanding of classes. Indeed, most set theorists object to taking proper classes seriously because set theory is our most general theory of collections. We shall not go into this issue in any further depth here, but will return to the idea of NBG in section 5 .

This, then, is how we interpret Cantor's famous dictum:

The Absolute can only be acknowledged, but never known, nor even approximately known. (Cantor 1932: 205)

We cannot have a rational (mathematical) understanding of God; but we do have a rationally compelling argument establishing the existence of God.

We now turn our attention to Premise 1, which requires further defence. We have established that it is reasonable to claim that God exceeds human understanding, but not explicitly that anything that

${ }^{19}$ Or even the stronger class theory Morse-Kelly (MK). 
exceeds human understanding is an aspect of God. We will not offer any sort of formal argumentation to this conclusion here; however, the next subsection will offer some defence of such a claim.

\subsubsection{The Last Step}

Cantor himself was happy to take the last step - i.e. to regard the (humanly incomprehensible) proper classes as part of something that fundamentally and maximally transcends us: God.

Asfar as the discussion here has gone, the arguments we are considering do seem to establish (if they are sound) the existence of a completed set theoretic universe that, as an entity, fundamentally transcends human understanding - and there is a venerable tradition of identifying God with that which maximally transcends human understanding. ${ }^{20}$ Before considering more thoroughly whether such a tradition is justified, it is worth briefly considering whether there are other phenomena (within the mathematical sphere) which also surpass human understanding. If there are, considerable doubt would be shed upon Premise $1 .^{21}$

Two potential candidates for this are contradictions and (tokens of) the multiplication of huge numbers. We claim that neither example poses a real threat to our claims. For example, contradictions are not beyond human understanding because, instead of being unintelligible, they are simply necessarily false. The multiplication of extremely large numbers may exceed human understanding in practice (i.e. we could not practicably carry out such multiplications), but not in principle. We have the methods to carry them out and could carry them out whilst remaining finite beings; we would just need certain adjustments - for example, more memory space and longer life spans. The crucial point is that such adjustments would always leave us within the finite (and, correspondingly, human) sphere. Though we will not offer more discussion of this issue here, we believe all similar proposed counterexamples can be dealt with along the same lines. Therefore, we do not have an excess of examples of things that exceed human understanding and, as such, no initial doubt is cast on the claim that anything that exceeds human understanding is an aspect of God.

So, if it is true that God can be identified with that which maximally transcends human understanding, our arguments do seem to entail that

${ }^{20}$ See (Drozdek 1995).

${ }^{21}$ Thanks to Christian Tapp for drawing our attention to the need for this elucidation. 
$\mathrm{V}$ is somehow a part of the Divine. Nonetheless, it could be objected that such a conclusion tells us little else about the Divine. For example, it is a long way removed from establishing the existence of a personal God. Cantor himself saw his work as a means of bringing people to 'rational theism' (Letter to Hermite (1894), Cantor 1991: 124-125). But the argument that we have offered falls short of a theistic conclusion, and we see no means of adapting the argument to reach it. To establish this, something more would be needed (perhaps an appeal to the Bible).

In view of this, it might be argued that it is misleading to say that the arguments, taken together, establish the existence of God. This seems fair enough. Yet it is worth noting that the more minimalist conception of God adopted in this paper is somewhat in keeping with the properties attributed to potential divine entities in section 3. There we claimed that God exceeds human understanding and that, if anything were to be a fundamental characteristic attributable to a divine entity, that would be it. It would be unusual (though of course not impossible) for something else to possess a quality fundamental to God, and thus it seems compelling to suggest that anything that surpasses human understanding is (at least a part of) God. This is - again - not a formal or watertight defence of our decision to identify God with that which transcends human understanding, but we feel that it at least goes some way to establishing such an identification's legitimacy.

It is also worth mentioning another way in which our 'God' diverges from the traditional conception. This is that, upon our account, we have no assurance of God's 'goodness'; to put it in a (perhaps oversimplified) way, the divine entity our arguments, if sound and valid, would establish could just as viably be 'Satan' as God. However, this is not an issue for us. As our caveat at the end of the introduction stated, we use 'God' to refer to any sort of divine entity. As a result, such a differentiation is not relevant to us.

\subsubsection{Why Care?}

Let us for the moment reject the objections to the soundness of the arguments presented and suppose that our proposed responses to them are accepted. In this case, it seems that the arguments must be basically sound. The arguments in themselves are certainly not very complicated, and so it seems that something like them must have occurred to theologians, philosophers, mathematicians, and indeed maybe even to Cantor himself, at some point. Indeed, at least in embryonic form, the 
argument that we have tried to spell out in some detail has appeared in the literature:

Cantor joins Augustine in theological pronouncements and - as far as mathematics allows him - he also shows that it is a mathematically proven fact. There is no set of all sets, the number of infinities surpasses any number. This fact can be used by theologians [to show] that God simply must surpass all infinities and in this sense he is not infinite - he is the Absolute. (Drozdek 1995: 139)

This leads to the question of why such arguments have not been investigated and discussed in any detail before. We consider briefly why this might be the case, but claim that they are nonetheless still of merit especially for philosophers.

The most likely reason that theologians have not been interested in such argumentation is that - as articulated before - the existence of a personal God is not established by it - and a personal God would be of the most interest to them.

Mathematicians (and in particular set theorists) are only interested in what is subject to the laws of mathematics. V, at least as far as the arguments at hand are concerned, falls outside the scope of this, even if its existence has to be acknowledged to some extent in mathematical practice. As such, strictly speaking, conclusions that can be derived from a consideration of $\mathrm{V}$ are outside the remit of their interests.

Cantor himself did not articulate such arguments. The reason seems to be that for him platonism about sets (Premise 1) is derived from prior premises of a philosophical and / or theological nature:

[...] a relation analogous to that between theology and metaphysics can be ascertained to hold between on the one hand the latter (metaphysics) and on the other hand mathematics and the other natural sciences. The foundation of the principles of mathematics and the natural sciences is the responsibility of metaphysics; she must therefore regard them both as her children and as her servants and helpmates, which she cannot afford to lose out of sight but instead must always guard and control, and which must produce from the wide scope of the material and mental realm the building blocks with which her palace can be completed, like the queen bee residing in her apiaries sends out thousands of bees into the garden to suck nectar from the flowers and then together and under her supervision transform it into honey. (Cantor, Letter to Esser (1896), Tapp 2005: 308-309, our translation) 
It is for this reason that Cantor takes recourse to ideas from Augustinian theology. In other words, he rejects the mathematical naturalistic stance that has become popular since the work of philosophers such as Gödel, Quine, Putnam and others and, in essence, works backwards from theism to platonism. We, in contrast to Cantor, take platonism to be grounded in mathematical practice. More precisely, it can be inferred from mathematical practice by inference to the best explanation (essentially an indispensability argument). ${ }^{22}$ Cantor would also have objected to our minimalist conception of God: he upheld the idea of a personal God and in fact rebelled vehemently when he was charged with pantheism (Letters to Franzelin (1886), Cantor 1991: 254-258), and he also objected to deism. He insisted on a conception of God that does not leave deism or pantheism viable possibilities.

In light of this, it is important not to lose sight of the fact that, if the premises hold, the arguments do establish something. They are noncircular: the existence of something that exceeds the limits of human intelligibility is derived rather than presupposed. As such, it seems that philosophers at least should be interested: philosophers follow arguments where they lead as long as the conclusions are significant, even if they fall short of what theologians are interested in. Henceforth, we shall refer to the philosophical position which we have adopted as the 'naïve stance' (for obvious reasons), and shall give reasons for preferring it to arguably more sophisticated views.

\section{THE NAÏVE STANCE}

We have seen that from certain 'sophisticated' philosophical perspectives, one or more of our premises can be challenged. We have not refuted the challenges from these perspectives, and nor do we think we could: the perspectives are wholly coherent. Nonetheless, there are reasons for preferring the naïve naturalistic attitude that we have adopted in this article.

Firstly, as we saw earlier, the platonism that it involves is the ontological stance that mathematicians implicitly adopt in their practice. It is also heuristically immensely fruitful, as is universally acknowledged. Even the 'working mathematician' who does not, upon reflection, endorse the platonistic stance that she adopts in her mathematical working hours

${ }^{22}$ For a discussion of indispensability arguments in the philosophy of mathematics, see (Colyvan 2011). 
will recognise that it is in practice impossible to do mathematics without adopting the naïve stance.

Secondly, one can formulate a class theoretic reflection principle that expresses that $\mathrm{V}$ is ineffable in a very specific sense (Welch and Horsten 2012). The principle in question states, roughly, that $\mathrm{V}$ is second order elementary equivalent to some set sized initial segment $V_{\kappa}{ }^{23}$ in the sense that every second-order formula is true in $\mathrm{V}$ with all its proper classes if and only if it is true in the structure $\left\langle\mathrm{V}_{\mathrm{K}}, \mathrm{V}_{\mathrm{K}+1}, \varepsilon\right\rangle$. This principle is called the Global Reflection Principle (GRP). It can be shown that if GRP is added to a weak (set theoretic conservative) class theory such as a fragment of NBG, strong large cardinal consequences follow. ${ }^{24}$ At the same time, the theory NBG+GRP can be shown to be set theoretically sound from certain large cardinal axioms (the axiom of subcompact cardinals). Thus it seems right to say that the mathematically Absolutely Infinite is mathematically unknowable in a very fundamental sense. ${ }^{25}$ And this class theoretic reflection principle can only be formulated against the background of a class theory (such as NBG) in which $\mathrm{V}$ is recognised to exist. Thus we can extend the scope of the indispensability argument from sets to proper classes. The outcome is that support is given to the claim that the set-theoretic universe exists as a mereological whole of all individual sets.

The global reflection principle is a principle about V. We have argued that it is true. Thus it could be objected that it seems that, after all, it is possible for us to have some mathematical (rational) knowledge of $\mathrm{V}$ after all; as such, it would be an exaggeration to say that we can have no knowledge about the about the Absolutely Infinite at all. However, GRP does say that a certain kind of knowledge can never be had of V. We have to acknowledge that it is not a set, but at the same time we cannot know any proposition of it that, when relativized to any initial segment $V_{\alpha}$ would be false. This is enough, we submit, to claim that $\mathrm{V}$ fundamentally exceeds human comprehension.

The derivation of these large cardinal consequences (i.e. the existence of unboundedly many Woodin cardinals) from GRP is currently the only way of providing intrinsic support for them. But it seems that GRP is most straightforwardly motivated against the background of

${ }^{23} \mathrm{~V}_{\mathrm{k}}$ is the set of those sets of ordinal rank smaller than $\mathrm{k}$.

${ }^{24}$ In particular, NBG+GRP entails the existence of unboundedly many Woodin cardinals.

${ }^{25}$ This argumentative move is an instance of Inference to the Best Explanation. 
an actualist platonist stance about sets and proper classes. ${ }^{26}$ This is, we submit, testimony to the fruitfulness of the naïve stance that we have adopted in this article.

\section{MATHEMATICS, METAPHYSICS, AND THEOLOGY}

The initial part of this paper was dedicated to supporting the claim that, contrary to popular opinion, Cantor had a largely coherent and defensible view of how the set theoretic universe as a whole should be considered. His conception of such a universe was not restricted to the mathematical sphere; for Cantor, the domains of discourse of mathematics and theology were thoroughly intertwined:

[...] the unbreakable bond that connects metaphysics with theology; where on the one hand the latter is the lodestar to which the former directs itself and from which it receives its light, when the natural and ordinary lights fail; on the other hand, theology needs for its scientific development and for its representation the whole of philosophy, which therefore stands to it in a subservient relationship. This has three consequences: a), that theology inevitably is a participant in any metaphysical discussion; b), that any real progress in metaphysics also strengthens or multiplies the tools of theology, indeed, in certain circumstances can lead human reason [...] with respects to mysteries of faith to deeper, more contentful symbolic insight than could have been expected or suspected beforehand. (Letter to Esser (1896), Tapp 2005: 308, our translation)

Moreover, Cantor, contrary to what we would say today, classifies his own work in set theory as metaphysics (in a subsequent letter to Esser from 1896):

The general theory of manifolds [...] belongs entirely to metaphysics. You can easily convince yourself from this, when you examine the categories of cardinal number and ordinal type, these fundamental concepts of set theory, with respect to the degree of their generality and besides will remark, that in them thought is completely pure, so that there is not even the least scope for the imagination to play a role. This is not altered in the least by the images, to which I, like all metaphysicians, from time to time help myself, and also the fact that the works of my pen are published in

${ }^{26}$ An attempt to motivate GRP without assuming that the concept of set and of proper class are instantiated is given in (Welch and Horsten 2012: section 7). 
mathematical journals, does not modify the metaphysical content and character of them. (Tapp 2005: 309-310, our translation)

This leaves the reader of Cantor's works with a mixture of mathematical, metaphysical and theological elements that are related to each other in complicated and at times confusing ways. For this reason, it is not easy to find a coherent rational argumentative structure in Cantor's views. Nonetheless, we have attempted to do this, paying especial attention to his characterisation of the mathematical universe, his conception of the divine, and the similarities between the two.

We then attempted to use Cantor's insights into the parallels between the Absolutely Infinite and God to construct a rational argument for the existence of some sort of divine entity. Whereas Cantor worked from a theological standpoint to mathematical platonism, we have attempted to motivate a theological standpoint upon the assumption that mathematical platonism is correct. Our arguments, if sound, establish the existence of a divine entity - but crucially fall short of establishing the personal 'God' one might hope for. The 'God' that they establish is more akin to a pantheistic one, identified to some extent (or in part) with the mathematical universe.

We acknowledge that our arguments rely heavily on the acceptance of controversial premises and concepts. Mathematical platonism is often especially unpalatable to philosophers; the Cantorian actualist conception of the mathematical universe is unpalatable to many mathematicians and philosophers; a minimalist conception of God (compatible with deism or pantheism) is unsatisfactory to most theologians. Nonetheless, we believe that the arguments we have proposed are interesting and fruitful avenues to go down, whoever you are and whether you agree with them or not.

\section{BIBLIOGRAPHY}

Augustine of Hippo. 2003. The City of God (St. Ives: Penguin Classics) Balaguer, M. A. 1995. 'Platonist Epistemology', Synthese, 103: 303-325

Benacerraf, P. \& H. Putnam (eds.). 1983. Philosophy of Mathematics: Selected Readings, Second edition (Cambridge: Cambridge University Press)

Benacerraf, P. 1983. 'Mathematical Truth', reprinted in (Benacerraf and Putnam 1983: 403-420)

Bernays, P. 1983. 'On Platonism in Mathematics', in (Benacerraf and Putnam 1983: 258-271)

Burgess, J. \& G. Rosen. 1999. A Subject With No Object: Strategies for Nominalistic Interpretation of Mathematics (Oxford: Oxford University Press) 
Cantor, G. 1932. Abhandlungen Mathematischen und Philosophischen Inhalts. Herausgegeben von Ernst Zermelo (Berlin: Verlag Julius Springer)

Meschkowski, H. \& W. Nilson (eds.). 1991. Georg Cantor (Briefe: Springer)

Colyvan, M. 2011. 'Indispensability Arguments in the Philosophy of Mathematics', Stanford Encyclopedia of Philosophy, available at: $<\mathrm{http}: / / \mathrm{www}$. science.uva.nl/ seop/entries/mathphil-indis/> [accessed 20/09/2013]

Drake, F. 1974. Set Theory: Introduction to the Theory of Large Cardinals (Amsterdam: North-Holland)

Drozdek, A. 1995. 'Beyond Infinity: Augustine and Cantor', Laval Théologique et Philosophique, 51: 127-140

Hallett, M. 1984. Cantorian Set Theory and Limitation of Size (Oxford: Clarendon Press)

Hauser, K. 2011. 'Cantor's Absolute in Metaphysics and Mathematics' (Unpublished manuscript)

Hellman, G. 1989. Mathematics Without Numbers (Oxford: Oxford University Press)

Jané, I. 1995. 'The Role of the Absolutely Infinite in Cantor's Conception of Set', Erkenntnis, 42: 375-402

Jané, I. 2010. 'Idealist and Realist Elements in Cantor's Approach to Set Theory', Philosophia Mathematica, 18: 193-226

Kowalewski, G. 1950. Bestand und Wandel. Meine Lebenserinnerungen zugleich ein Beitrag zur neueren Geschichte der Mathematik (Munich: Oldenburg)

Martin, D.A. 2001. 'Multiple Universes of Sets and Indeterminate Truth Values', Topoi, 20: 5-16

Murawski, R. 2010. 'Cantor's Philosophy of Set Theory', in R. Murawski, Essays in the Philosophy and History of Logic and Mathematics (Amsterdam: Rodopi), pp. 15-28

Niebergall, K.-G. 2012. 'Mereology', in L. Horsten \& R. Pettigrew (eds.) Continuum Companion to Philosophical Logic (London: Continuum), pp. 271-298

Tapp, C. Kardinalität und Kardinäle. Franz Steiner, 2005.

Tapp, C. 2012. 'Absolute Infinity: A Bridge Between Mathematics and Theology?' To appear in: N. Tennant (ed.) Foundational Adventures: Essays in Honor of Harvey M. Friedman, available at: <http://foundationaladventures.com/> [accessed 25/09/2013]

Van Inwagen, P. 1994. 'Composition as Identity', Philosophical Perspectives, 8: $207-220$

Welch, P. \& L. Horsten. 'Absolute Infinity' (Submitted for publication)

Zermelo, E. 1996. 'On Boundary Numbers and Domains of Sets', translated by M. Hallett, in W. Ewald, From Kant to Hilbert: A Source Book in Mathematics. Volume 2 (Oxford: Oxford University Press), pp. 1208-1233 